\title{
Towards an Adaptive Defuzzification: Using Numerical Choquet Integral
}

\author{
Vicenç Torra $^{1(\bowtie)}$ and Joaquin Garcia-Alfaro ${ }^{2}$ \\ 1 Hamilton Institute, Maynooth University, Maynooth, Ireland \\ vtorra@ieee.org \\ 2 Institut Polytechnique de Paris, CNRS SAMOVAR, Télécom SudParis, France \\ jgalfaro@ieee.org
}

\begin{abstract}
Fuzzy systems have been proven to be an effective tool for modeling and control in real applications. Fuzzy control is a well established area that is used in a large number of real systems. Fuzzy rule based systems are defined in terms of rules in which the concepts that define the rules (both in the antecedent and consequent) can be defined in terms of fuzzy sets. In applications, rules are fired and then a set of consequents need to be combined to make a final decision. This final decision is often computed by means of a defuzzification method. In this paper we discuss the defuzzification proces and propose the use of a Choquet integral for this process. In contrast with standard defuzzification methods which are based on mean operators (usually discrete), the Choquet integral permits us to have an output variable with values that have different importances and with interactions among the values themselves. To illustrate the approach, we use a numerical Choquet integral software for continuous functions that we have recently developed. We also position the application of the approach to handle the uncertainty associated to a mission-oriented Cyber-Physical System (CPS).
\end{abstract}

\section{Introduction}

Knowledge based systems are used in a large number of real-world applications. Among them, rule based systems stand out for their interpretability. As the name indicates, they are defined in terms of sets of rules, with each rule defined by an antecendent that establishes when the rule applies, and a consequent that establishes the conclusion when the rule applies. Different types of rules have been considered. We want to underline the case of rules that permit to represent some type of uncertainty.

Fuzzy rules $[5,10]$ are the rules to be used when we need to consider vagueness and fuzziness in the concepts (either in the antecedent or the consequent). Recall that a concept on the reference set $X$ is imprecise when different elements of $X$ satisfy the concept (e.g., temperature below zero) and a concept is vague when there are values in $X$ for which it is doubtful to affirm that they satisfy or not the concept (e.g., temperature is cold). Vague and imprecise concepts can be represented by means of fuzzy sets.

(C) Springer Nature Switzerland AG 2019

V. Torra et al. (Eds.): MDAI 2019, LNAI 11676, pp. 113-125, 2019.

https://doi.org/10.1007/978-3-030-26773-5_11 
In contrast to standard (crisp) sets where characteristic functions are defined in terms of the Boolean sets $\{$ true, false $\}$ or $\{0,1\}$ (i.e., an element is either in a set $A$ or not), fuzzy sets are defined in terms of membership functions that assign to each element a value in the $[0,1]$ interval. So, for a set $A$ on the reference set $X$, a characteristic function for $A$ is typically of the form $\chi_{A}: X \rightarrow\{0,1\}$ while a membership function is of the form $\mu_{A}: X \rightarrow[0,1]$. Then, a value $\mu_{A}(x)$ of zero means no membership, a value of one means total membership, and values in $(0,1)$ represent partial membership.

Fuzziness distinguishes from probability because while a fuzzy membership of e.g. 0.7 represents partial truth, probability of 0.7 is a measure of our certainty of being the fact completely true. To illustrate the difference [3] gives an example of two bottles $A$ and $B$ where $A$ is marked with membership of 0.91 to be potable and $B$ with probability 0.91 to be potable. The later means that $B$ will be potable $91 \%$ of the trials, but $9 \%$ may be deadly. In contrast, the fuzzy bottle $A$ will contain perfectly potable water, maybe not completely pure, but not deadly in any case.

Due to the fact that the rules are defined using fuzzy sets, and fuzzy sets can be partially satisfied, at a given time, several rules may apply. This is so because each of the rules may satisfy partially the conditions given in the antecedent. The degree of satisfaction of a rule (the truth value of the antecedent) is computed taking into account the fuzzy sets that define the rules, as well as appropriate operators (e.g., operators to model conjunction and disjunction of the concepts in the antecedent). Fuzzy systems usually fire all rules with positive degree of satisfaction. Then, this degree is propagated to the conclusion. This leads to a set of conclusions that need to be considered together, with each conclusion having the corresponding degree of satisfaction of the rule.

A variety of fuzzy rule based systems exist in the literature. A detailed discussion of their differences is beyond the interest of this paper. See, for instance, references $[5,8-10,15,16,21]$ for a detailed description of some of them. We will focus on rule based systems in which the terms of both antecedents and consequents are described in terms of fuzzy sets. For simplicity, we will consider antecedents in which terms are only combined in a conjunctive way, and in which consequents have only one variable. Observe that this constraint is not relevant in our work because we focus on how to operate with the conclusions of the rules. Therefore, rules follow this pattern:

\section{If $V_{1}$ is $T_{1}$ and $V_{2}$ is $T_{2}$ and $\ldots$ and $V_{2}$ is $T_{2}$ then $V_{O}$ is $T_{O}$}

We will give a more accurate description of the rules in Sect. 2 . Here, $V_{i}$ represent variables, $T_{i}$ terms that are represented by fuzzy sets, $V_{O}$ is the output variable and $T_{O}$ an output term.

In our study, the output of a rule will be a fuzzy set. This fuzzy set will be defined in terms of the fuzzy set $T_{O}$ of a rule, and the degree of satisfaction of the antecedent. The collection of fuzzy sets obtained from all rules need to be combined (through a data fusion or aggregation process). We obtain in this way an aggregated fuzzy set. In order to obtain this set, we can proceed in 
different ways. In this work we will use the union of sets, as commonly used in the literature [5].

The combination of consequents of rules does not lead to an appropriate output. Most applications in control and modeling need as output a single value in the appropriate range of the output variable (e.g., the actual value to give to a controller). Therefore, a fuzzy set as system's output cannot be used without further processing. Defuzzification is the name of the process to select a single value from the aggregated fuzzy set. Different defuzzification strategies have been defined in the literature. The center of area is probably the most used one, but other solutions have been proposed.

Most defuzzification procedures can be seen as a two step process (see e.g. [6]). First, the aggregated fuzzy set is transformed into an appropriate distribution (e.g., a probability distribution via normalization or a distribution that eliminates inappropriate values). Then, an element is selected from the distribution (e.g., the expected value of the distribution).

In this paper, we propose the use of a Choquet integral [4] in the defuzzification process. This permits us to take into consideration some particularities of the domain of the output variable. For example, we may consider that different subdomains of the output variable have different relevance, and that different subdomains are incompatibles.

In a CPS, physical and software components are tightly connected. Realtime decisions are often needed, and systems need to adapt dynamically to their context. Rule-based approaches are appropriate for reactive systems. Changes in the environment cause changes in the input variables, that are quickly propagated into the output. Fuzzy rule based systems are an effective approach for implementing reactive systems.

Our model for defuzzification based on the Choquet integral permits us a dynamic modification of the focus of interest of the output variable. While keeping constant the fuzzy rule based system and the aggregation process, we will be able to adapt the center of interest of the output variable taking into account some additional variables of the environment. This will be done by means of a modification of the parameter of the Choquet integral (i.e., the fuzzy measure). As it is shown later, we consider an example with a simulated annealing-type approach where the defuzzification shifts from a less supported value to a more supported one (more conservative) when time progresses.

The structure of the paper is as follows. In Sect. 2 we give an overview of fuzzy measures and the Choquet integral. We will use these concepts later to formally define our approach. In Sect. 3 we describe the type of fuzzy rule based system we use in this work. In Sect. 4 we formalize our defuzzification method. In Sect. 5, we position the application of the approach to handle the uncertainty associated to a mission-oriented CPS. The paper finishes with some conclusions and lines for future work. 


\section{Fuzzy Measures and the Choquet Integral}

Let us start reviewing the concept of fuzzy measure, also known as non-additive measure or monotonic game. Fuzzy measures generalize additive measures and probabilities replacing the additivity condition by a condition on monotonicity: monotonicity with respect to set inclusion.

Definition 1. Let $(\Omega, \mathcal{F})$ be a measurable space. A set function $\mu$ defined on $\mathcal{F}$ is called a fuzzy measure if an only if

- $0 \leq \mu(A) \leq \infty$ for any $A \in \mathcal{F}$;

$-\mu(\emptyset)=0$;

$-\mu(\Omega)=1$;

- If $A_{1} \subseteq A_{2} \subseteq \mathcal{F}$ then

$$
\mu\left(A_{1}\right) \leq \mu\left(A_{2}\right)
$$

The boundary condition $\mu(\Omega)=1$ is not always required. In our context, it will be convenient. It corresponds to the condition that the probability of the whole reference set $\Omega$ is one.

When the set of reference is discrete, the Choquet integral of a function with respect to a fuzzy measure is defined as follows.

Definition 2. Let $X$ be a reference set, and let $\mu$ be a fuzzy measure on $X=$ $\left\{x_{1}, \ldots, x_{n}\right\}$; then, the Choquet integral of a function $f: X \rightarrow \mathbb{R}^{+}$with respect to $\mu$ is defined by

$$
\text { (C) } \int f d \mu=\sum_{i=1}^{n}\left[f\left(x_{s(i)}\right)-f\left(x_{s(i-1)}\right)\right] \mu\left(A_{s(i)}\right),
$$

where $f\left(x_{s(i)}\right)$ indicates that the indices have been permuted so that $0 \leq$ $f\left(x_{s(1)}\right) \leq \cdots \leq f\left(x_{s(n)}\right) \leq 1$, and where $f\left(x_{s(0)}\right)=0$ and $A_{s(i)}=$ $\left\{x_{s(i)}, \ldots, x_{s(n)}\right\}$.

When the reference set is not finite, the Choquet integral of $f$ with respect to $\mu$ is defined with the following expression

$$
\text { (C) } \int f d \mu:=\int_{0}^{\infty} \mu_{f}(r) d r
$$

where $\mu_{f}(r)=\mu(\{x \mid f(x) \geq r\})$.

This definition generalizes the Lebesgue integral, and it reduces to the Lebesgue integral when $\mu$ is additive.

\section{$3 \quad$ Fuzzy Rule Based Systems}

We will consider fuzzy rules following the structure below. For the sake of simplicity, we consider that all rules have $n$ input variables and a single output. 
As we have indicated in the introduction, we only allow for conjunction to combine the variables and their terms in the antecedent.

$\mathrm{R}$ : If $V_{1}$ is $t_{1 a}^{R}$ and $V_{2}$ is $t_{2 b}^{R}$ and $\cdots V_{n}$ is $t_{n z}^{R}$ then $Y$ is $t_{y o}^{R}$

Here, $V_{i}$ represents an input variable and $X_{i}$ its domain, $t_{i e}^{R}$ is a term for the $i$ th input variable, $Y$ represents an output variable and $t_{y o}^{R}$ is a term for output variable. More specifically, for each variable $X_{i}$, we have a set of terms $n_{i}$ terms denoted by $t_{i 1}, \ldots, t_{i n_{i}}$. Similarly, we have a set of $n_{o}$ terms for the output variable $Y$. These terms are $t_{y 1}, \ldots, t_{y n_{o}}$. Naturally, $t_{1 a}^{R} \in\left\{t_{11}, \ldots, t_{1 n_{1}}\right\}$, $t_{2 b}^{R} \in\left\{t_{21}, \ldots, t_{2 n_{2}}\right\}$, etc.

Each $t_{i j}$ is described in terms of a fuzzy set defined on the domain of variable $V_{i}$. We denote this fuzzy set by $\mu_{i j}$. Naturally, $\mu_{i j}: X_{i} \rightarrow[0,1]$.

Let us consider the rule below for controling the temperature of a device. We have two input variables $\epsilon$ and $\Delta \epsilon$ representing, respectively, the error (with the objective temperature) and error difference (change in the error in two consecutive time instants), and an output variable that controls the device. We have three terms, one for each variable. They are, positive, positive, and small-negative. Each term will be defined by a fuzzy set. Fuzzy sets will be defined in the range of the variable (this means that the term positive for variable $\epsilon$ may be different than the term positive for variable $\Delta \epsilon$ ).

\section{Rule1: if $\epsilon$ is positive and $\Delta \epsilon$ is positive then control-variable is small-negative}

Fuzzy inference for fuzzy rule based systems for this type of rules usually follows four steps. We give these fours steps formally below. We begin with an informal description based on the previous rule.

Step 1. Rules are fired when we have actual values for each of the input variables. E.g., we have that the error $\epsilon$ is 3 degrees $(\epsilon=3)$ and that the error has decreased 0.2 degrees $(\Delta \epsilon=-0.2)$. Then, the degree of satisfaction for each variable is defined as the membership degree of the actual value of the variable using the appropriate membership function. In our case $\mu_{\epsilon \text {, positive }}(3)$.

In our rules, the only operator in the antecedent is and. Because of that, the membership degrees obtained for each variable in the antecedent are combined using an operator that models the conjunction. In fuzzy logic, t-norms play this role. The minimum $\min (a, b)$ and the product $a \dot{b}$ are examples of t-norms. This combination corresponds to the degree of satisfaction of the antecedent.

Step 2. The degree of satisfaction of the antecedent is propagated to the consequent. This is done clipping the membership function associated to the output variable using the degree of satisfaction of the antecedent. The process implies that when the antecendent is completely satisfied (i.e., degree equal to one), the output is just the fuzzy term. In contrast, when the antecedent is not at all satisfied (i.e., degree equal to zero), the output is just the empty set (i.e., all membership values are zero).

Step 3. All rules of our knowledge base (say $K B$ ) are fired applying the approach just described. We obtain in this way a collection of fuzzy sets (say $\mu_{R}$ for each rule $R$ in $K B$ ). All these fuzzy sets are combined. It is usual to use the 
union of all fuzzy sets for this purpose. We obtain in this way an aggregated fuzzy set. From a mathematical point of view, this approach is to consider fuzzy rules in a disjunctive way. That is, either we apply $R_{1}$ or $R_{2}$ or $\ldots$ or $R_{t}$. Under this interpretation, we take the union of the outputs (the output of $R_{1}$ or the output of $\left.R_{2} \ldots\right)$. For crisp and disjoint rules, this would result into a single output. In the case of fuzzy rules, this step results into a fuzzy set that contains pieces of information of several rules. See e.g. Figure 1 that shows a typical output of Step 3. It corresponds to the union of two clipped fuzzy sets (one with maximum value at $y=2$ and the other with maximum value at $y=3)$. Then, in Step 4 we obtain a kind of average of the outputs (average weighted by the degree of satisfaction of the rule).

In a previous work [18] we showed that the process of combination can be expressed in terms of a Sugeno integral [14]. Understanding the fusion of consequents in this way, we generalize the usual approach introducing a model in which rules do not need to be independent. A similar idea is present in $[2,11]$ where the Choquet integral is used to combine outcomes from rules, also to permit non independent rules.

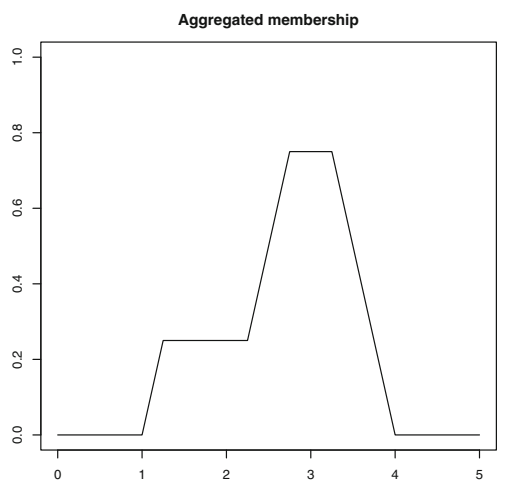

Fig. 1. Example of aggregated fuzzy set obtained in Step 3.

Step 4. A value is obtained from the aggregated set. This step is called defuzzification. The center of area is one of this defuzzification processes. This method is the one given in Step 4 below.

We give now a more formal definition of this process.

Step 1. Degree of satisfaction of the antecedent of each rule $R$

$$
\alpha^{R}=T\left(\mu_{1 a}^{R}\left(x_{1}\right), \mu_{2 b}^{R}\left(x_{2}\right), \ldots, \mu_{n}^{R}\left(x_{n z}\right)\right)
$$

for a t-norm $T$.

Step 2. Application of all rules $R$ and computation of their consequents

$$
\mu^{R}(x)=\min \left(\alpha^{R}, \mu_{y o}^{R}\right) .
$$


Step 3. Application of a set of rules and computation of the corresponding consequent. In terms of the membership function, it is the following:

$$
\tilde{\mu}=\cup_{R \in K B} \mu^{R} \text {. }
$$

If we consider memberships at given $y$ in the space of the output $Y$, this is

$$
\tilde{\mu}(y)=\cup_{R \in K B} \mu^{R}(y)
$$

for all $y \in Y$. We often use a t-conorm (e.g., the maximum) to compute the union.

Step 4. Defuzzification

$$
y^{*}=\frac{\sum_{y} \tilde{\mu}(y) \cdot y}{\sum_{z} \tilde{\mu}(z)} \text { or } y^{*}=\frac{\int y \tilde{\mu}(y) d y}{\int \tilde{\mu}(z) d z} .
$$

\section{Defuzzification Based on the Choquet Integral}

Equation 3 is one of the existing approaches to select a value from the aggregated fuzzy set. This approach is known as center of area or gravity defuzzification. Driankov et al. [5] describe five other methods: center of sums, center of largest area, first of maxima, middle of maxima, and height defuzzification.

As briefly explained in the introduction, following [6], most defuzzification procedures can be seen from a two step process perspective. The first step transforms the fuzzy set $\tilde{\mu}$ into an appropriate distribution, and the second step is about selecting an element from the distribution. In the center of area, the transformation is about building the following probability distribution from the fuzzy set $\tilde{\mu}: p(y)=\tilde{\mu}(y) / \sum_{z} \tilde{\mu}(z)$, or $p(y)=\tilde{\mu}(y) / \int \tilde{\mu} d z$ in the continuous case. Selection is defined as the expected value of the distribution $p$. First of maxima sets to zero all memberships that are not maximal and then selects the first $y$ with a maximal membership (i.e., let $\alpha=\sup _{z} \tilde{\mu}(z)$ then $\tilde{\mu}^{\prime}(y)=\alpha$ if and only if $\tilde{\mu}(y)=\alpha$ and select $\left.\min _{y}\left\{y \mid \tilde{\mu}^{\prime}(y)=\alpha\right\}\right)$. Middle of maxima follows the same approach but selects $0.5\left(\min _{y}\left\{y \mid \tilde{\mu}^{\prime}(y)=\alpha\right\}+\max _{y}\left\{y \mid \tilde{\mu}^{\prime}(y)=\alpha\right\}\right)$.

Mathematically, the two step process for the center of area corresponds to (i) building the probability distribution and then (ii) computing the expectation of this distribution. We use the definition of the expectation as the Lebesgue integral of the function $f(y)=y$ with respect to the probability distribution. That is, considering a continuous membership function $\tilde{\mu}$ :

$$
y^{*}=\int y d p
$$

where $p(y)=\tilde{\mu}(y) / \int \tilde{\mu}(z) d z$.

Our contribution is to use in this process the Choquet integral of the function $f(y)=y$ with respect to a measure $\nu$ built from the fuzzy set $\tilde{\mu}$. That is, our proposal is to defuzzify using

$$
y^{*}=(C) \int d \nu
$$


where $\nu$ is built from $\tilde{\mu}$. Therefore, we need to consider ways to construct fuzzy measures $\nu$ defined for all the sets $[x, \infty]$ (note that the values of $\nu([x, \infty])$ are the only ones actually considered in the integration process). We give some examples below. In the examples we use $p(y)=\tilde{\mu}(y) / \int \tilde{\mu}(y) d z$ as above.

Example 1. If we define $\nu([x, \infty])=\int_{x}^{\infty} p(x) d x$ then the Choquet integral corresponds to the Lebesgue integral, and the defuzzification is the center of area.

Example 2. If we define $\nu([x, \infty])=Q\left(\int_{x}^{\infty} p(x) d x\right)$ for a distortion function $Q$ (i.e., a function $Q(x)$ such that $Q(0)=0, Q(1)=1$ and monotonic with respect to $x$ ), we result into the continuous WOWA operator for defuzzification (see [17] for details). Note that measures of this form correspond to distorted probabilities. I.e., $\nu=Q o P$.
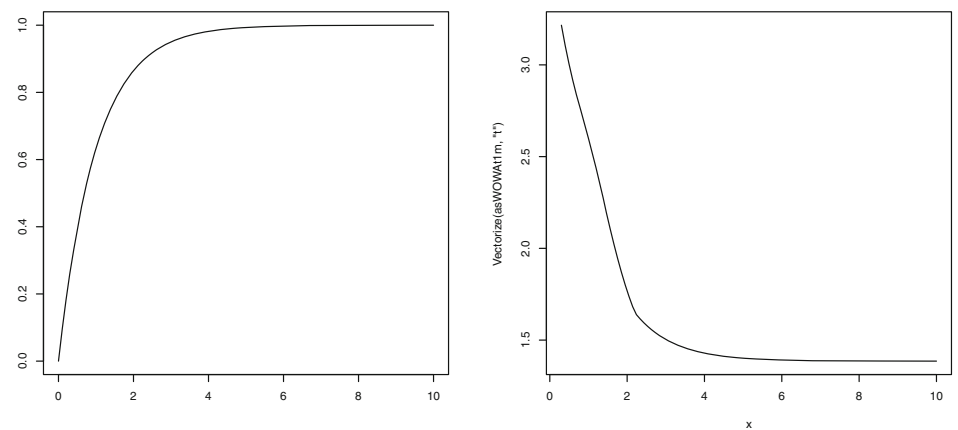

Fig. 2. Function $1-e^{-k}$ used to define the distortion function $Q_{k}$, and the resulting defuzzified values.

Let us consider the continuous piece-wise linear functions $Q_{(a, b)}(x)$ defined as 0 for $x<a, 1$ for $x>b$, and linear between $a$ and $b$. This is to model that the values that accumulate probabilities in $(a, b)$ (as in quantile functions) are the most relevant in the defuzzification process. Let us consider a system where we can consider risky alternatives (with less suport) at the initial times, but more conservative alternatives (with more support) at later times. To model this situation, we consider $t_{k}=e^{k}$ (a popular cooling schedule - the exponential one - in simulated annealing). We then consider the intervals $\left(a_{k}, b_{k}\right)$ defined as $\left(\max \left(0,1-e^{k}-0.1\right), \min \left(1,1-e^{k}+0.1\right)\right)$ to build the corresponding distortions $Q_{k}$. The function $1-e^{k}$ as well as the outcome of the defuzzification with respect to time $k$ is given in Fig. 2 (left and right, respectively).

We can see in Fig. 2 (right) how the defuzzified value tend to the minimum when time increases. 
Example 3. For a given fuzzy measure $\nu^{\prime}$, we define the measure $\nu$ by set $\tilde{\mu}$ :

$$
\nu([x, \infty])=\frac{(C) \int_{[x, \infty]} p d \nu^{\prime}}{K},
$$

where $p(y)$ is defined as before $p(y)=\tilde{\mu}(y) / \int \tilde{\mu}(z) d z$, and with normalizing factor $K=(C) \int_{[-\infty,+\infty]} p d \nu^{\prime}$. Note that in this equation, we can use equivalently either $\tilde{\mu}$ or $p$ because we have the normalizing factor $K$. For $\nu^{\prime}=\lambda$ (i.e., $\nu^{\prime}$ is the Lebesgue measure), this definition results into the measure above $\nu([x, \infty])=\int_{x}^{\infty} p(x) d x$. For fuzzy measures $\nu^{\prime}$, this results into a fuzzy measure $\nu$.

To illustrate this example, we have considered two cases: measures $\nu_{1}^{\prime}$ and $\nu_{2}^{\prime}$ which result using Expression 5 into two measures $\nu_{1}$ and $\nu_{2}$. They are the following ones:

$$
\begin{aligned}
& -\nu_{1}^{\prime}([a, b])=\left(l_{1} / 5\right)+\left(l_{2} / 5\right)^{2}+0.2\left(l_{1} / 5\right)\left(l_{2} / 5\right)^{4} \\
& -\nu_{2}^{\prime}([a, b])=\left(l_{1} / 5\right)+\sqrt{l_{2} / 5}+0.2\left(l_{1} / 5\right) \sqrt{\sqrt{l_{2} / 5}}
\end{aligned}
$$

where $l_{1}=\lambda([a, b] \cap[0,3])$ and $l_{2}=\lambda([a, b] \cap[3,6])$ (here $\lambda$ is the Lebesgue measure).

The first measure gives more relevance for elements $x<3$ than the second measure that have more relevance for elements $x>3$. Compare the measure of $\nu_{1}([2,3])=0.6373673$ and $\nu_{1}([3,4])=0.08365445$ vs. $\nu_{2}([2,3])=0.2400694$ and $\nu_{2}([3,4])=0.6262801$. This implies that the defuzzification using $\nu_{1}$ is smaller than the defuzzification using $\nu_{2}$. Experiments show that this is true, as in the first case we obtain a defuzzified value of 2.306174 while in the second case it is 2.576696 .

Example 3 shows that defuzzification using a Choquet integral provides additional flexibility, and that we can model situations in which different regions of the domain have different relevance. We can exploit non-additivity of the measure to reduce or increase the relevance of conservative or risky values. In addition, we can also consider measures in line with Example 2 where the measure is timedependent, and, thus permits us to shift the focus of the system over time even when its inputs do not change.

The outcomes of these examples have been computed considering the membership functions as continuous functions and computing the Choquet integrals numerically. The numerical Choquet integral has been computed using the software in $\mathrm{R}$ we provided in [20]. In Example 3, we need to compute the Choquet integral of $f(x)=x$ with respect to $\nu$ in the interval $[0,6] . \nu$ is the Choquet integral of $\tilde{\mu}$ with respect to $\nu$. The integration of $f$ requires the computation of $\nu([a, 5])$ for several values of $a$ (i.e., several numerical Choquet integrals). This makes the process costly. E.g., the defuzzification of the fuzzy set using $\nu_{2}$ takes $43 \mathrm{~s}$ in a Laptop (Intel(R) Core(TM) i7-8550U CPU @ $1.80 \mathrm{GHz}$ ). 


\section{Applying the Approach to Cyber-Physical Systems}

We present in this section the application of the defuzzification approach presented in this paper, to handle the uncertainty associated to a mission-oriented $\mathrm{CPS}^{1}$. We assume a CPS in which entities integrate computation, communication and physical processes [7]. Examples include robotics and autonomous vehicles. Our CPS scenario assumes the existence of a decentralized process that computes corrective control actions based on our defuzzification approach. Mathematically, the CPS is modeled as a spatially distributed system whose control loops are closed by a wireless communication network. The communication network connects the different components of the CPS, assumed to be a series of mobile agents that exchange messages to complete a mission-oriented problem. The scenario follows previous work presented in $[1,12]$ (cf. http://j. $\mathrm{mp} / \mathrm{scavesim}$ for further details and some related media), in order to address classical theoretical problems studied in the CPS literature, such as stabilization of mobile systems and control-theoretic techniques addressing uncertainty. A quick summary of the scenario is presented next.

\subsection{Trajectory Search Scenario}

A series of mobile agents (e.g., unmanned aerial vehicles) must accomplish a mission. The mission relies on a trajectory search scenario. The agents must cross different segments of, e.g., city blueprints, by physically identifying an unknown number of intermediary trajectory points in each segment. Identifying and visiting all the trajectory points, as well as reaching the final destination, it is crucial to label the mission as accomplished. The agents collect and deliver information at each trajectory point, such as taking pictures and exchanging messages between them, in order to discover the way to reaching the following trajectory point. The mission is considered as accomplished when all the intermediate points specified in the trajectory are successfully visited by all the agents. The mission fails when at least one of the agents fails at identifying or visiting one of the points in the trajectory.

A trajectory is represented as an unknown number of intermediate points. At every trajectory point, the agents must collectively determine the next step by solving a search problem [1]. We define the trajectory search problem as a collective solution in which a bounded number of agents follow a trajectory from $s$ (starting point) to $t$ (terminal point), as depicted in Fig. 3 .

The agents must discover the trajectory points instructed by a decentralized CPS process. The process is to collect the information from all the agents, and apply the defuzzification approach presented in this paper in the reasoning process. Together, the agents travel from a trajectory point to any other. With the trajectory points identified to vertices, they form a complete graph. We assume

\footnotetext{
${ }^{1}$ The term CPS, coined in 2006 by H. Gill at the National Science Foundation [13], refers to next generation embedded ICT systems, which include monitoring and control technologies in charge of physical components for pervasive applications.
} 


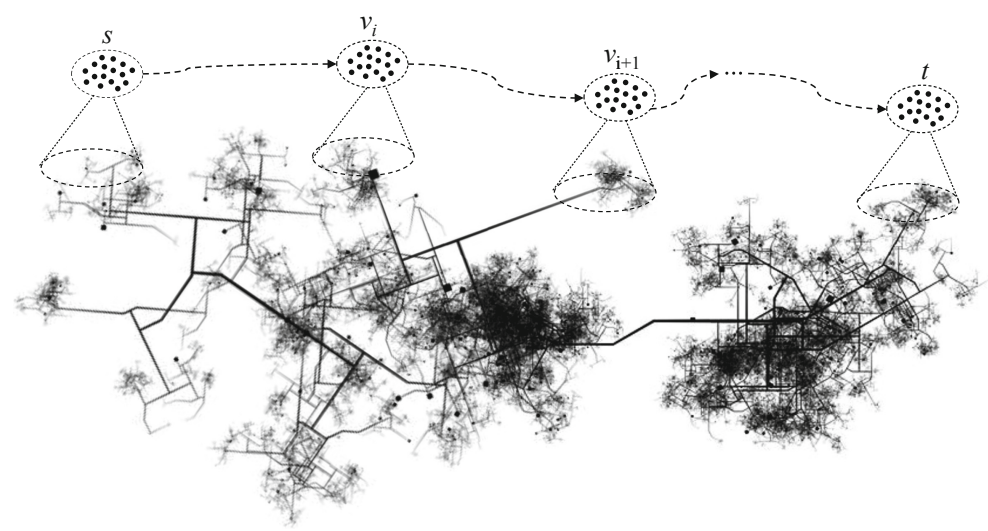

Fig. 3. Group of mobile agents traveling from $s$ (initial point of a trajectory) to $t$ (terminal point of the trajectory). Segments $\left(v_{i}, v_{i+1}\right)$ represent the connection of two trajectory points $\left(v_{i}\right.$ and $\left.v_{i+1}\right)$. They cooperate to navigate from $s$ to $t$.

that the agents are unaware of the terrain and locations of the trajectory points. However, their CPS process has the capability to correlate data collectively collected by the overall system and take decisions. The agents communicate and exchange information between them. The mission of the agents is assumed to be accomplished if all the agents successfully find all the trajectory points within the segments that start in $s$ and end in $t$. See [22] for further details.

\section{Concluding Remarks}

In this paper we have proposed the use of Choquet integrals in the defuzzification of fuzzy rule based systems. We have shown with some examples that our definition permits to take into account the relevance of different regions. We have also considered using the proposed approach for the accomplishment of critical missions under uncertain conditions of a mission-oriented CPS. We have presented a CPS scenario in which a series of mobile agents are instructed to solve a trajectory search problem. The agents must discover and physically visit a series of unknown trajectory points. To successfully accomplish the mission, all the agents must collectively share information at each trajectory point, such as taking pictures or exchanging messages between them, in order to discover the following steps towards the following step of the trajectory. The mission is said to have been accomplished when all the intermediate segments specified in the trajectory are successfully visited by all the agents. The mission fails when one of the agents fails at identifying or visiting one of the trajectory points. Future work includes embedding the approach in a fuzzy system, and develop more efficient solutions to compute the defuzzified values using the Choquet integral. 
Acknowledgments. Support from the European Commission, under grant agreement 830892 (H2020 SPARTA project), and the Cyber CNI Chair of the Institut MinesTélécom, supported by the Center of excellence in Cybersecurity, Airbus Defence and Space, Amossys, EDF, Nokia, BNP Paribas and the Regional Council of Brittany.

\section{References}

1. Barbeau, M., Garcia-Alfaro, J., Kranakis, E.: Geocaching-inspired resilient path planning for drone swarms. In: Joint 7th International Workshop on MissionOriented Wireless Sensor and Cyber-Physical System Networking (MiSeNet) and 12th International Workshop on Wireless Sensor, Robot and UAV Networks (WiSARN), co-located with IEEE INFOCOM: April-May 2019, France, Paris (2019)

2. Barrenechea, E., Bustince, H., Fernandez, J., Paternain, D., Sanz, J.A.: Using the choquet integral in the fuzzy reasoning method of fuzzy rule-based classification systems. Axioms 2, 208-223 (2013)

3. Bezdek, J.C.: Fuzzy models - what are they, and why? IEEE Trans. Fuzzy Syst. 1, 1-1993 (1993)

4. Choquet, G.: Theory of capacities. Ann. Inst. Fourier 5, 131-295 (1953/54)

5. Driankov, D., Hellendoorn, H., Reinfrank, M.: An Introduction to Fuzzy Control. Springer, Heidelberg (1993). https://doi.org/10.1007/978-3-662-11131-4

6. Filev, D., Yager, R.R.: A generalized defuzzification method under BADD distribution. Int. J. Intel. Syst. 6(7), 687-697 (1991)

7. Kim, K., Kumar, P.: Cyber-physical systems: a perspective at the centennial. Proc. IEEE 100, 1287-1308 (2012)

8. Magdalena, L.: Hierarchical fuzzy control of a complex system using metaknowledge. In: Proceedings of the IPMU 2000 Conference, pp. 630-637 (2000)

9. Magdalena, L.: On the role of context in hierarchical fuzzy controllers. Int. J. Intell. Syst. 17(5), 471-493 (2002)

10. Ross, T.J.: Fuzzy Logic with Engineering Applications, 4th edn. Wiley, Hoboken (2016)

11. Sanz, J., Lopez-Molina, C., Cerrón, J., Mesiar, R., Bustince, H.: A new fuzzy reasoning method based on the use of the Choquet integral. In: Proceedings of the EUSFLAT (2013)

12. Shi, W., Garcia-Alfaro, J., Corriveau, J.-P.: Searching for a black hole in interconnected networks using mobile agents and tokens. J. Parall. Distrib. Comput. 74(1), 1945-1958 (2014)

13. Song, H., Rawat, D., Jeschke, S., Brecher, C.: Cyber-Physical Systems: Foundations, Principles and Applications. Elsevier, Amsterdam (2016)

14. Sugeno, M.: Theory of Fuzzy Integrals and its Applications, Ph.D. Dissertation, Tokyo Institute of Technology, Tokyo, Japan (1974)

15. Takahagi, E.: On fuzzy integral representation in fuzzy switching functions, fuzzy rules and fuzzy control rules. In: Proceedings of the 8th IFSA Conference, pp. 289-293 (1999)

16. Torra, V.: A review on the construction of hierarchical fuzzy systems. Int. J. Intell. Syst. 17(5), 531-543 (2002)

17. Torra, V., Godo, L.: Continuous WOWA operators with application to defuzzification. In: Calvo, T., Mayor, G., Mesiar, R. (eds.) Aggregation Operators, pp. 159-176. Physica-Verlag, Heidelberg (2002). https://doi.org/10.1007/978-3-79081787-4_4 
18. Torra, V., Narukawa, Y.: The interpretation of fuzzy integrals and their application to fuzzy systems. Int. J. Approx. Reasoning 41(1), 43-58 (2006)

19. Torra, V., Narukawa, Y., Miyamoto, S.: Modeling decisions for artificial intelligence: theory, tools and applications. In: Torra, V., Narukawa, Y., Miyamoto, S. (eds.) MDAI 2005. LNCS (LNAI), vol. 3558, pp. 1-8. Springer, Heidelberg (2005). https://doi.org/10.1007/11526018_1

20. Torra, V., Narukawa, Y.: Numerical integration for the Choquet integral. Inf. Fusion 31, 137-145 (2016)

21. Tunstel, E., Oliveira, M.A.A., Berman, S.: Fuzzy behavior hierarchies for multirobot control. Int. J. Intell. Syst. 17, 449-470 (2002)

22. http://www-public.imtbs-tsp.eu/ garcia_a/web/prototypes/scave/ 